\begin{tabular}{ccc}
\hline & International Journal of Engineering \& Technology, $7(3.33)(2018) 124-127$ \\
SPC & International Journal of Engineering \& Technology \\
Website $:$ www.sciencepubco.com/index.php/IJET & Research paper \\
\hline
\end{tabular}

\title{
Gait Imbalance Determination Using Foot Plantar Pressure Measurement
}

\author{
Myung Jae Lim', Yun Jin-Nam², Dong Kun Chung ${ }^{3}$, Young Man Kwon ${ }^{3} *$ \\ ${ }^{I}$ Department of Medical IT, College of Healthy Industry, Eulji University, Korea \\ e-mail:lk04@eulji.ac.kr \\ ${ }^{2}$ Department of Medical IT, College of Healthy Industry, Eulji University, Korea \\ e-mail :kdo10000@naver.com \\ ${ }^{3}$ Department of Medical IT, College of Healthy Industry, Eulji University, Korea \\ e-mail:ymkwon@eulji.ac.kr \\ *Corresponding author E-mail: ymkwon@eulji.ac.kr
}

\begin{abstract}
The ability to walking is a basic component of human mobility, and gait, which is the pattern of walking, may help or harm human health depending on posture. Therefore, it is a part of smart health care to measure gait quality in real time and inform the user of the posture. This paper determines whether body balance is maintained during gait by using foot plantar pressure measurement among methods to determine the gait balance. Thus, it aims to investigate changes in body balance according to the weight of bags and the plantar pressure measurement system, and to implement a system that give a notice when an unbalanced gait occurs in the future study.
\end{abstract}

Keywords: Pressure measurement, Foot pressure, Walking analysis, Healthcare, Pedar-x system.

\section{Introduction}

Since the gait is the basis of all activities, Walking is something that the average person probably doesn't give much thought. It's our most basic method of transportation, but an inability to walk or be mobile can drastically change a person's life. It can impact our independence and also create significant health problems over both the short and long term.

walking with proper posture helps to improve health through the balance maintenance and alignment of the body, whereas walking with improper posture leads to physical imbalance and body fatigue due to ineffective use of muscles and joints, resulting in postural deformities and gait disabilities[1,2].

If a person walks with a weighted bag, the weight of the bag causes stress on his or her body, and a change occurs in dynamic balance and posture due to the mechanical and physiological effects. This may cause the problems of the nervous system such as peripheral nerve damage, and the musculoskeletal system such as injury to shoulder and arm muscles, spinal deformity, back pain and fatigue of plantar intrinsic foot muscles[3,11].

The weight of the bag may lead to abnormal gait patterns and cause diseases such as scoliosis, especially for middle, high school and college students carrying various books and supplies in their bags. Therefore, this paper seeks to measure changes in foot plantar pressure according to the method of carrying bags and to identify whether carrying bags breaks the balance of the body to propose a solution system for resolving problems caused by carrying bags[4,12].

\section{Related Study}

\subsection{Kinematic Gait Analysis System}

Kinematics describes the gait from the perspectives of body segment, joint acceleration and velocity, angel and position (dislocation), and the kinematic system is used in the gait analysis to record the location and direction of the body segment, the angle of joints, and the corresponding linear velocity, angular velocity and acceleration.

The kinematics describes only the temporal and spatial indices, which are the general characteristics of gait, and the motion of the torso and lower limbs during gait, without providing information about the related forces. However, both kinematic and kinetic data are needed for the proper quantitative description of complex motor activities such as gait[4,14].

\subsection{Kinetic Gait Analysis System}

Kinetics is a field for studying forces, moments, masses and accelerations without taking into account the location and orientation of the objects involved. For example, a device known as a force platform is used to measure the force under the foot during gait without information about the position of a lower limb or the angel of a joint. In connection with gait kinetics, the important use of the force platform is to measure muscle activity by calculating the joint moments[5,15]. 


\subsection{Pressure Unit Pascal (Pa)}

$1 P \alpha=1 \frac{\mathrm{N}}{\mathrm{m}^{2}}=1 \frac{\frac{\mathrm{kg} \cdot \mathrm{m}}{\mathrm{s}^{2}}}{\mathrm{~m}^{2}}=1 \frac{\mathrm{kg}}{\mathrm{m} \cdot \mathrm{s}^{2}}=1 \frac{\mathrm{kg}}{100 \mathrm{~cm} \cdot \mathrm{s}^{2}}=\frac{1}{100} \cdot \frac{\mathrm{kg}}{\mathrm{cm} \cdot \mathrm{s}^{2}}$

Pascal $(\mathrm{Pa})$ is the SI derived unit of pressure measurement. One pascal corresponds to the pressure when a force of one newton per square meter is applied. In the preceding equation, $\mathrm{N}$ is the newton, $\mathrm{m}$ is the meter, $\mathrm{kg}$ is the kilogram, and $\mathrm{s}$ is the time(second)[5,9].

\subsection{Kinematic and Kinetic Validity}

Movements of the whole-body center of mass during quiet standing have been estimated from measurements of body segment movements. These whole-body center of mass movements have been compared with movements of the center of mass as predicted from a simple inverted-pendulum model of standing. However, the total body center of mass is a weighted average of the center of mass of all individual body segments. The question arises as to how well the total body center of mass represents the individual segments and lower limb joint angles. This study focuses on the validity of how well the individual segments and lower limb angles temporally and spatially synchronize with the total body center of mass.

Segment center of mass root-mean-square displacements were strongly correlated with center of mass height relative to the ankle joint and were synchronized, or temporally locked, to the movement of the whole body center of mass. Sagittal plane ankle angular displacements were highly correlated to sagittal plane center of mass movement; stronger correlations between body center of mass and lower limb angular displacement were observed, the result of compensatory knee joint angular displacements. These data support and extend the use of an inverted pendulum model to represent quiet standing postural control[4].

\subsection{Body Kinematic}

Human quiet stance is often modeled as a single-link inverted pendulum pivoting only around the ankle joints in the sagittal plane. However, several recent studies have shown that movement around the hip joint cannot be negligible, and the body behaves like a double-link inverted pendulum. The purpose of this study was to examine how the hip motion affects the body kinematics in the sagittal plane during quiet standing. Ten healthy subjects were requested to keep a quiet stance for 30 s on a force platform. The angular displacements of the ankle and hip joints were measured using two highly sensitive CCD laser sensors. By taking the second derivative of the angular displacements, the angular accelerations of both joints were obtained.

As for the angular displacements, there was no clear correlation between the ankle and hip joints. On the other hand, the angular accelerations of both joints were found to be modulated in a consistent anti-phase pattern. Then we estimated the anterior-posterior (A-P) acceleration of the center of mass (CoM) as a linear summation of the angular acceleration data. Simultaneously, we derived the actual CoM acceleration by dividing A-P share force by body mass. When we estimated CoM acceleration using only the angular acceleration of the ankle joint under the assumption that movement of the CoM is merely a scaled reflection of the motion of the ankle, it was largely overestimated as compared to the actual CoM acceleration.

Whereas, when we take the angular acceleration of the hip joint into the calculation, it showed good coincidence with the actual CoM acceleration. These results indicate that the movement around the hip joint has a substantial effect on the body kinematics in the sagittal plane even during quiet standing[6,7 ].

\section{Foot Plantar Pressure Measurement System}

\subsection{System Configuration}

The novel pedar-x measurement system calculates the forces acting on the insole by the pressure unit pascal and displays the results with graphics, graphs and figures on the monitor.

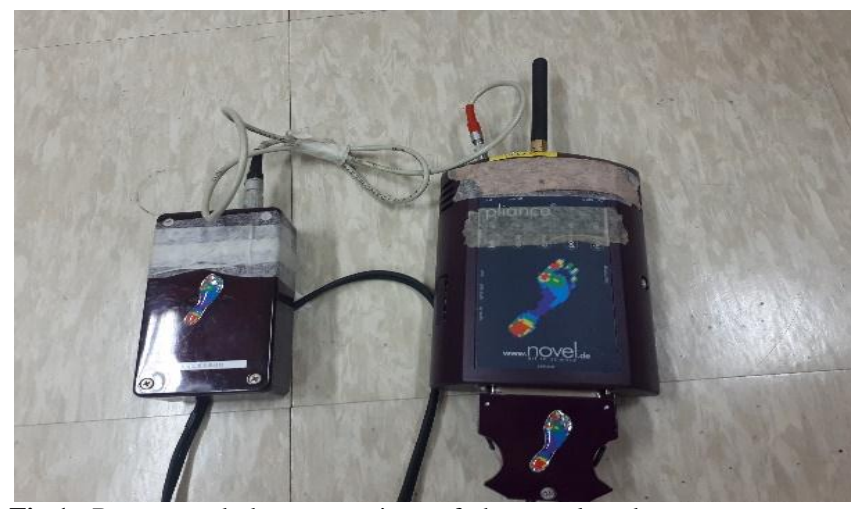

Fig.1: Battery and data transmitter of the novel pedar-x measurement system.

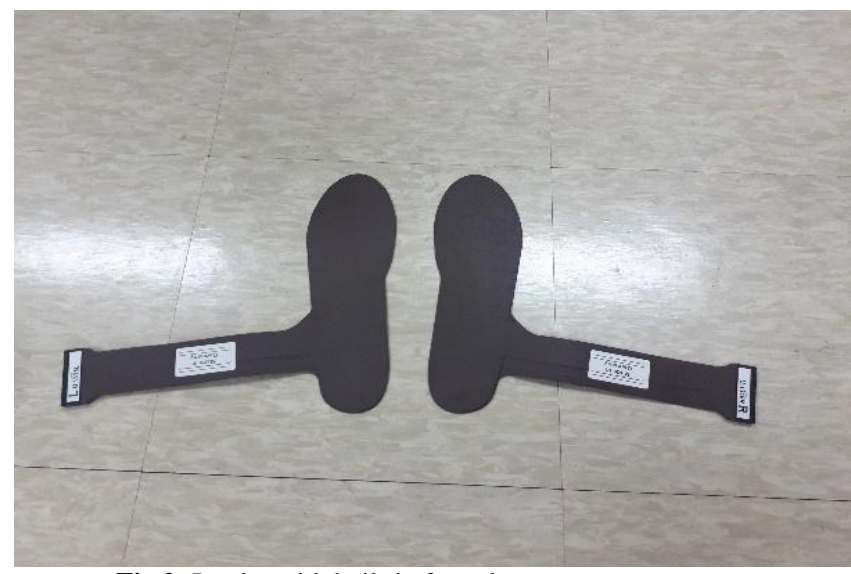

Fig.2: Insoles with built-in foot plantar pressure sensors

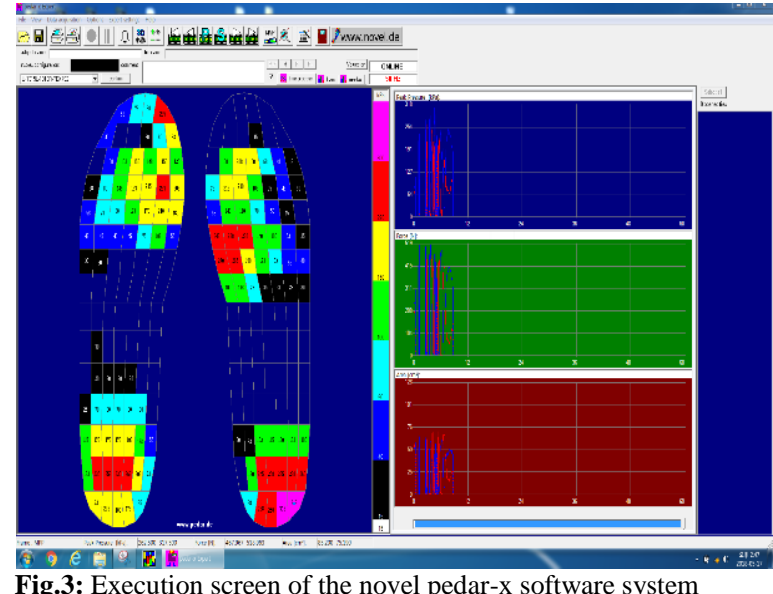

The pedar® system is a pressure distribution measurement system for monitoring the regional loading between the foot and the shoe. It works with the Bluetooth wireless telemetry system and can also function in a mobile capacity as it communicates with various Bluetooth PC adapters. The pedar ${ }^{\circledR}$ system is connected to the sensor pads on the dorsal, medial or lateral areas of the foot covering the entire plantar surface, and it allows multiple synchronization options to use with EMG and video systems for gait analysis The functions and features of the pedar ${ }^{\circledR}$ software are summarized in the following table [5]. 
Table1: Functions and features of the pedar software

\begin{tabular}{|c|c|}
\hline Functions & Features \\
\hline $\begin{array}{l}\text { - Acquisition and storage of dy- } \\
\text { namic pressure distribution data } \\
\text {-View of absolute pressure values } \\
\text { in each sensor and gaitline } \\
\text {-Presentation and playback of } \\
\text { dynamic measurement } \\
\text { - View of maximum pressure, force } \\
\text { and contact area } \\
\text { - Maximum pressure picture (MPP) } \\
\text { - Mean value picture (MVP) } \\
\text {-Printout of both insole and pro- } \\
\text { duction in original size } \\
\text { - Storage of collected data with } \\
\text { comments } \\
\text { - Color } 2 \text { d display of pressure data }\end{array}$ & $\begin{array}{l}\text { - Individual sensor selection } \\
\text { - Online and offline modes } \\
\text { - Numeric display of pressure pic- } \\
\text { ture } \\
\text { - Animation of foot contact phases } \\
\text { - Step timing analysis } \\
\text { - Gait comparison and difference } \\
\text { pictures } \\
\text { - Calculation of regional loadings } \\
\text { - ASCII output }\end{array}$ \\
\hline
\end{tabular}

Table2: Technical data for pedar insoles Technical data for pedar insoles:

\begin{tabular}{|l|l|}
\hline shoe size & 22 to 49 (European), 3 widths \\
\hline thickness $(\mathrm{mm})$ & $1.9(\mathrm{~min} .1)$ \\
\hline number of sensors & $85-99$ \\
\hline pressure range $(\mathrm{kPa})$ & $15-600$ or $30-1,200$ \\
\hline hysteresis $(\%)$ & $<7$ \\
\hline resolution $(\mathrm{kPa})$ & 2.5 or 5 \\
\hline offset temperature drift $(\mathrm{kPa} / \mathrm{K})$ & $<0.5$ \\
\hline minimal bending radius $(\mathrm{mm})$ & 20
\end{tabular}

Table 3: Technical data for pedar-x system Technical data for pedar-xf system:

\begin{tabular}{ll}
\hline dimension (mm) & $150 \times 100 \times 40$ \\
\hline weight $(\mathrm{g})$ & 400 \\
\hline number of sensors (max) & $256(1,024)$ \\
\hline measurement frequency & 20,000 sensors/second \\
\hline storage type & 2 GB SD card \\
\hline computer interface & fiber optic/USB and Bluetooth \\
\hline operating system & current Windows OS \\
\hline sync option & fiber optic/TTL, in and out/wireless \\
\hline power supply & NIMh battery
\end{tabular}

\subsection{Measurement Environment}

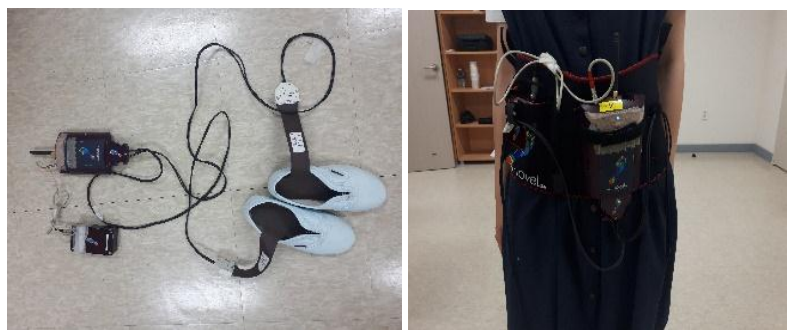

Fig.4: Experimental equipment and wearing the experimental equipment

The insoles with 99 built-in sensors on each side were inserted into the shoes, and the battery and transmitter connected to the insoles were fixed to the front of the stomach. Experiments were conducted by dividing the methods of carrying bags into three cases where no bag was worn, a backpack was worn on both shoulders, and a shoulder bag was only on the right shoulder in order to investigate the influence of loads imposed by carrying bags on gait and gait imbalance through the measurement of foot plantar pressures according to different bag-carrying methods. The weight of $3 \mathrm{~kg}$ mass was added to the weight of the backpack and shoulder bag, assuming the weight of the college textbooks and notebooks carried by general college students.

\subsection{Measurement Results}
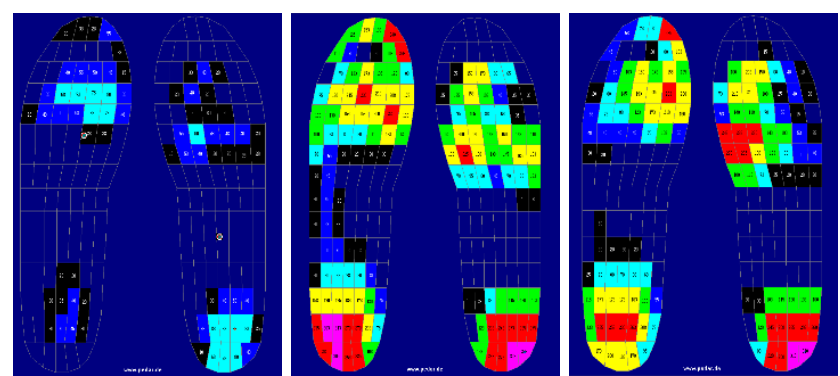

Fig 5: Foot plantar pressure measurement results in $2 \mathrm{~d}$ view

The figure shows a part of the measurements obtained from walking without wearing a backpack, walking with a backpack on both shoulders, and walking with a shoulder bag on the right shoulder. As shown in the figure, both feet are divided into the 99 parts of a grid on both sides, respectively, and the color changes according to the applied pressure. The color black (15-39) represents the lowest pressure value, followed by the light blue color (40-49), the mint color (50-99), the green color (100-149), the yellow color (150-219), the red color (220-299), and the pink color (more than 300 ), and the unit is $\mathrm{kPa}$.

Table 4: Foot plantar pressure measurement results (unit: $\mathrm{kPa}$ )

\begin{tabular}{|c|c|c|c|c|}
\hline \multicolumn{2}{|c|}{} & $\begin{array}{c}\text { Walking } \\
\text { without } \\
\text { wearing a } \\
\text { bag }\end{array}$ & $\begin{array}{c}\text { Walking after } \\
\text { wearing a } \\
\text { backpack }\end{array}$ & $\begin{array}{c}\text { Walking } \\
\text { after wear- } \\
\text { ing a shoul- } \\
\text { der bag }\end{array}$ \\
\hline \multirow{2}{*}{$\begin{array}{c}\text { Mean } \\
\text { value }\end{array}$} & Left foot & 172.590 & 196.629 & 178.414 \\
\cline { 2 - 5 } & Right foot & 224.426 & 233.278 & 289.916 \\
\hline $\begin{array}{c}\text { Maximum } \\
\text { value }\end{array}$ & Left foot & 443.695 & 469.455 & 467.967 \\
\cline { 2 - 5 } & Right foot & 463.340 & 511.203 & 518.963 \\
\hline
\end{tabular}

As shown above, the foot plantar measurement results were obtained by measuring the plantar pressures three times, respectively in each environment and using the results where most data were collected due to the least number of Bluetooth connection cut-outs.

$$
\text { Mean Value (unit : } \mathrm{kPa} \text { ) }
$$

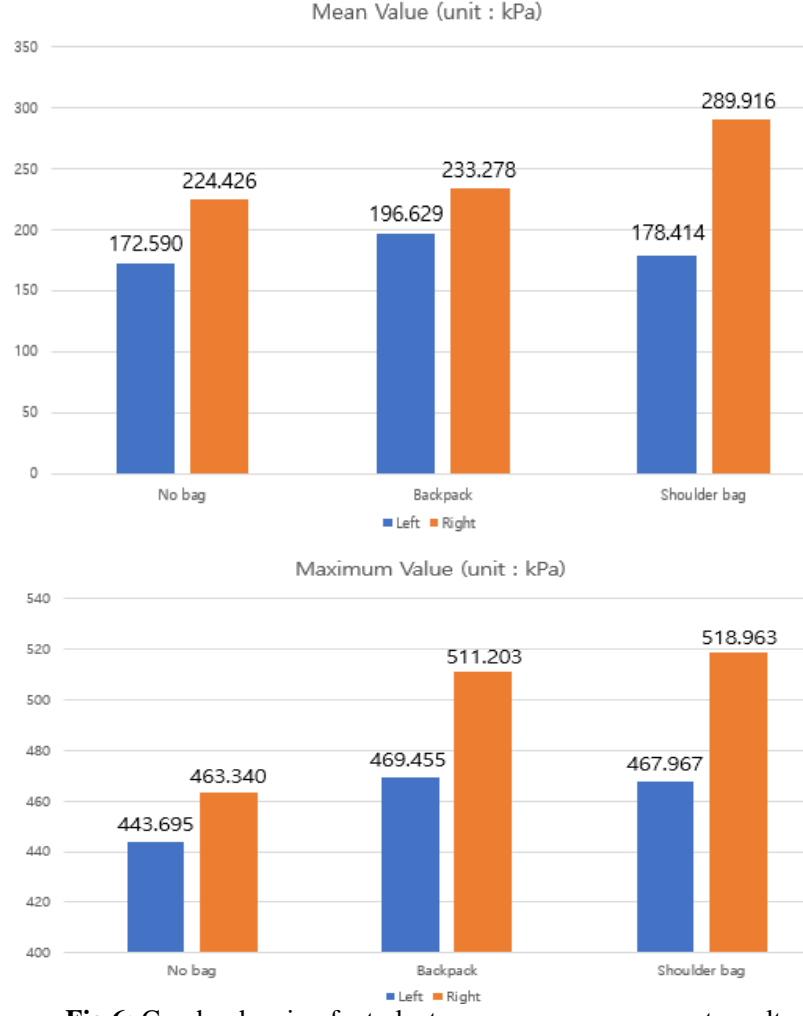

Fig.6: Graphs showing foot plantar pressure measurement results 
As shown above, the foot plantar measurement results were obtained by measuring the plantar pressures three times, respectively in each environment and using the results where most data were collected due to the least number of Bluetooth connection cut-outs. Based on the mean and maximum values of the results measured in 0.02 second units, higher pressure was found in the case of walking while wearing a bag than in the case of walking without wearing a bag, and the foot plantar pressure on the right foot was higher when a shoulder bag was worn on the right shoulder than when a backpack was worn on both shoulders. This demonstrates that the additional weight placed on the shoulder results in gait imbalance.

\section{Conclusion and Future Study Plan}

In this paper, a method to infer gait changes through the measurement of foot plantar pressures was selected among the methods that can be used to determine gait imbalance. Through this study, it was possible to enhance the understanding of the system which can measure the foot plantar pressures, and to confirm the foot planter pressure levels that vary depending on the pressure applied to the body due to the bag-wearing style. On the basis of the results, it was predicted that changes in body balance would occur as the foot plantar pressure levels changed according to the pressure of the bag on the body. Based on the findings of this study, the future research should implement a system to give a notice when the foot plantar pressure exceeding the predetermined level is measured, or when the difference of the foot plantar pressure between both feet is large.

\section{Acknowledgement}

The project has been possible due to the collaboration of Fine tech water CO., Ltd and Seoungnam Senior Experience Complex 's staff and students as well as many other institutions, companies, individuals and colleagues.

\section{References}

[1] asagawa S1, Ushiyama J, Kouzaki M, Kanehisa H. Effect of the hip motion on the body kinematics in the sagittal plane during human quiet standing. Neurosci Lett. 2009 Jan 23;450(1):27-31. doi: 10.1016/j.neulet.2008.11.027. Epub 2008 Nov 17

[2] Nami-Yun, "A Comparative Study on Walking Analysis by Age in Korea", The Graduate School of Seonam University(Department of Physical Therapy), 2010.

[3] Wkdkpedia 'Pa (unit)' https://ko.wikipedia.org/wiki/\%ED\%8C\%8C\%EC\%8A\%A4\%EC\% B9\%BC_(\%ЕB\%8B\%A8\%EC\%9C\%84)

[4] http://novel.de/novelcontent/pedar

[5] http://novel.de/novelcontent/software/pedar-software-list00

[6] Choi-JaeHyuk, "Hybrid Indoor Positioning Technology Research based on Pedestrian Dead Reckoning, Map Matching and Wireless Signal", Kwangwoon university graduate school electronic engineering, 2016

[7] Park-Ji Hye, "Study on the Indoor Positioning Using BLE Beacon", Kwangwoon university graduate school electronic engineering, 2016

[8] Choi-EuYeol, "Status of Location Information Technology and Utilization Cases", Korea Regional Information Development Institute, 2016

[9] http://naver.me/5gznrla6

[10] http://terms.naver.com/entry.nhn?docid=3571963\&cid=59088\&cat egoryid $=59096$

[11] Jin-Jo Cheoi, "Introduction to Status Awareness System Development Trends", Ninety system, p.3-4 , 2008

[12] D. Solomonow-Avnon, A. Wolf, A. Herman, N. Rozen, A. Haim, "Reduction of frontal-plane hip joint reaction force via mediolateral foot center of pressure manipulation: A pilot study", Orthop Res, doi: 10.1002/jor.22744, $2014 . \quad$ DOI: http://dx.doi.org/10.1002/jor.22744
[13] [2] B. M. Nigg, "Biomechanics of Running Shoe Champaign", IL: Human Kinetics; 1986.

[14] [3] S. J. Park, "The Analysis the Changes of Foot Pressure by Various Carrying a Pack Methods during Walking", The Graduate School of Health and Science Daegu University, Master's thesis, 2008.

[15] [4] Gage WH1, Winter DA, Frank JS, Adkin AL. Kinematic and kinetic validity of the inverted pendulum model in quiet standing. Gait Posture. 2004 Apr;19(2):124-32. pub med 